\title{
Une banque de données pour le calcul de barrage
}

\author{
A data bank for dam computation
}

\author{
N. NEDJAT \\ Chercheur, EDF-CNEH ${ }^{*}$, Ecole Centrale Paris ${ }^{\circ}$ \\ J.-J. FRY \\ Ingénieur d'études et développement hydraulique à EDF-CNEH ${ }^{*}$
}

Rev. Franç. Géotech. n 60, pp. $71-81$ (juillet 1992)

\section{Résumé}

L'objet de cet article est la définition des domaines de variation d'un certain nombre de paramètres mécaniques concernant les différents matériaux des barrages en enrochement. L'outil de base est une banque de données qui collationne entre autres les paramètres de DUNCAN. Les résultats trouvés restent en accord avec ceux déjà connus en mécanique des sols et ils permettent également de suggérer des résultats qui peuvent donner matière aux réflexions futures.

\footnotetext{
Abstract

This paper points out the fields of variation of some typical mechanical parameters used to described rockfill dams'materials. A data bank collecting various parameters, in particular DUNCAN parameters, serves as a basis for the present report. Naturally, the discovered results are in harmony with the usual knowledge of soils mechanics, but furthermore, these results suggest other results which open new prospects for futures studies.
}

- Centre national d'équipement hydraulique, 73373 Le Bourget Cedex.

* Ecole centrale des arts et manufactures, 92295 Châtenay-Malabry Cedex. 


\section{INTRODUCTION}

Le projeteur confronté dès l'avant-projet à la conception d'un ouvrage, sans avoir la possibilité de connaître l'ensemlbe des propriétés du remblai et de sa fondation est amené à justifier son projet à partir d'un calcul paramétrique. L'expérience montre que ce calcul est plus rapide et plus représentatif si les paramètres sont issus d'études antérieures sur des ouvrages similaires. La variété des situations incite alors à emmagasiner l'expérience acquise sous forme de banque de données.

Une banque de données concernant principalement les barrages en enrochement regroupe plus de 170 grands barrages dans le monde (décrits pour la plupart dans les comptes rendus de la CIGB) ; 750 matériaux constitutifs y sont répertoriés ; 124 paramètres définissent le comportement des matériaux et du barrage.

Une première exploitation succinte est présentée ciaprès. L'accent est mis sur le lien entre les paramètres physiques d'identification (densité, granulométrie) et les paramètres mécaniques issus de la loi de DUNCAN. Cette dernière a été choisie en raison notamment de l'utilisation internationale intensive qui nous a permis de retrouver un volume conséquent de données.

\section{RAPPEL CONCERNANT LA LOI DE DUNCAN}

La première formulation de cette loi est due à KONDNER (1963) qui a proposé que la courbe triaxiale contrainte déviatoire fonction de la déformation axiale soit approximée par l'hyperbole:

$$
\sigma_{1}-\sigma_{3}=\frac{\epsilon_{1}}{a+b \epsilon_{1}}
$$

où :

$$
a=\frac{1}{E_{1}} \text { et } b=\frac{1}{\left(\sigma_{1}-\sigma_{3}\right)_{\text {ult }}}
$$

avec :

$$
\mathrm{R}_{\mathrm{f}}=\frac{\left(\sigma_{1}-\sigma_{3}\right)_{\mathrm{ult}}}{\left(\sigma_{1}-\sigma_{3}\right)_{\mathrm{f}}}
$$

et $\quad\left(\sigma_{1}-\sigma_{2}\right)_{\mathrm{f}}$ : déviateur maximal,

La formulation complète de cette loi est donnée par DUNCAN en 1970. Les paramètres sont définis de la manière suivante.

Le module initial est donné par la relation :

$$
E_{1}=K P a\left(\frac{\sigma_{3}}{P a}\right)^{n} \text { où : } \mathrm{Pa}=0,1 \mathrm{MPa}
$$

Un module tangentiel est obtenu en dérivant l'équation de l'hyperbole:

$$
\mathrm{Et}=\mathrm{KPa}\left(\frac{\sigma^{3}}{\mathrm{~Pa}}\right)^{\mathrm{n}} \mathrm{X}^{2}
$$

où :

$$
X=1-\frac{\operatorname{Rf}\left(\sigma_{1}-\sigma_{3}\right)(1-\sin \Phi)}{2 c \cos \Phi+2 \sigma_{3} \sin \Phi}
$$

avec

$$
\begin{array}{lll}
\mathrm{c} & \vdots & \text { cohésion } \\
\Phi & \vdots & \text { angle de frottement } \\
\sigma_{3} & \text { et } & \sigma_{1} \\
\mathrm{~K} & \mathrm{n} & \text { contraintes principales } \\
\mathrm{n} & \text { coefficients sans unité }
\end{array}
$$

Le coefficient de Poisson est défini par une autre relation hyperbolique entre la déformation axiale $\epsilon_{1}$ et la déformation radiale $\epsilon_{3}$ :

$$
\epsilon_{1}=\frac{\epsilon_{3}}{F+D \epsilon_{3}}
$$

Les coefficients de Poisson initial $\nu_{1}$ et tangentiel $\nu_{\text {of }}$ sont définis de la même manière par:

$$
\nu_{1}=\mathrm{G}-\mathrm{F} \log _{10}\left(\frac{\sigma_{3}}{\mathrm{~Pa}}\right)
$$

et :

$$
\nu_{\mathrm{t}}=\frac{\mathrm{G}-\mathrm{Flog}_{10}\left(\frac{\sigma_{3}}{\mathrm{~Pa}}\right)}{\left(1-\mathrm{D}\left(\sigma_{1}-\sigma_{3}\right) / \mathrm{K} \mathrm{Pa}\left(\sigma_{3} / \mathrm{Pa}\right)^{n} \mathrm{X}\right)^{2}}
$$

avec: G, F, D : coefficients sans dimension.

Et enfin, l'angle de frottement peut suivre une variation suivant la contrainte de confinement:

$$
\Phi\left(\sigma_{3}\right)=\Phi-\Delta \Phi \log _{10}\left(\frac{\sigma_{3}}{\mathrm{~Pa}_{\mathrm{a}}}\right)
$$

Les paramètres principaux sont ceux qui caractérisent :

- la résistance : $\Phi$ (angle de frottement pour 0,1 $\mathrm{MPa}$ de confinement) et $\Delta \Phi$ (décroissance de l'angle de frottement entre 0,1 et $1 \mathrm{MPa}$ ) ;

- le module $\mathrm{K}$ (sans unité, mais en pratique équivalent au module d'YOUNG pour $0,1 \mathrm{MPa}$ puisque si on prend $\mathrm{Pa}=\sigma_{3}=0,1 \mathrm{MPa}$ dans la formule de $E_{i}$, on obtient $E=K$ en $0,1 \mathrm{MPa}$ ) et $n$ (exposant de la loi d'accroissement du module fonction de la contrainte).

\section{CLASSES DE MATÉRIAUX}

\subsection{Typologie étudiée}

Un remblai en terre est composé de matériaux aux fonctions bien distinctes comme le précise le tableau 1. Par conséquent, une première exploitation de la banque consiste à rechercher le comportement moyen, typique de chaque classe. La description granulométrique de chaque matériau est synthétisée par la figure 1 qui représente les principales classes en fonction de la taille de la portion fine $\left(\log _{10}(\mathrm{Cu})\right.$, avec $\mathrm{CU}=\mathrm{d}_{60} / \mathrm{d}_{10}$ où $\mathrm{d}_{60}$ est le diamètre de grain correspondant à $60 \%$ de passant en poids).

Il apparaît que l'étalement de la granulométrie augmente avec la finesse du matériau. Ainsi on note une évolution continue de l'enrochement au drain, puis au filtre jusqu'au sol de noyau. Les matériaux de transition recouvrent, bien sûr, les filtres et les drains. 


\section{BARRAGES EN ENROCHEMENT}

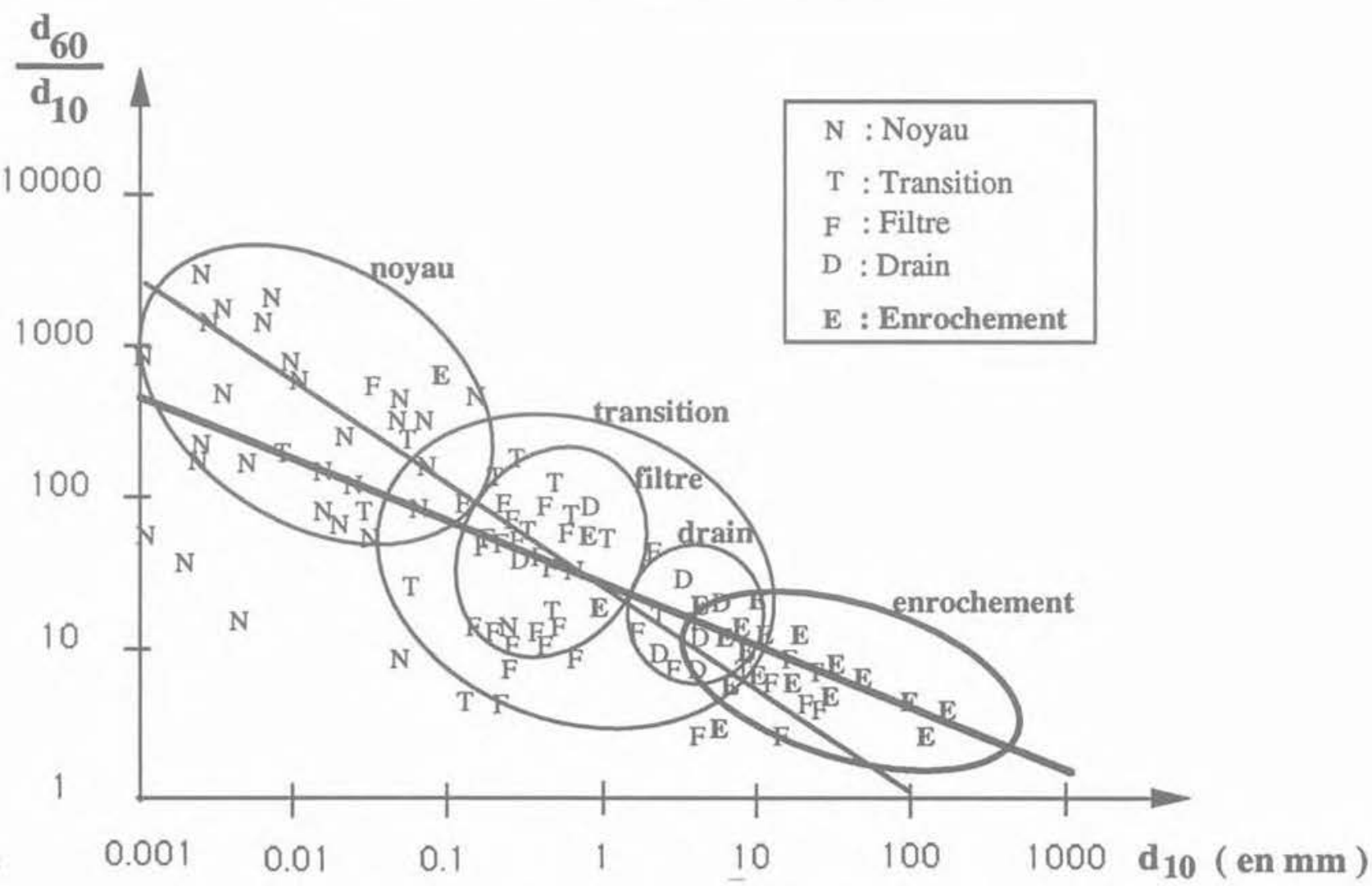

Fig. 1. - Variation de $d_{60} d_{10}$ en fonction de $d_{10}$ (en échelle logarithmique).

Tableau 1. - Typologie des matériaux constitutifs d'un remblai en terre.

\begin{tabular}{|c|c|}
\hline Fonction & Matériau \\
\hline Etanchéité & noyau \\
Lutte anti-érosion & filtre \\
Drainage & drain \\
Stabilité & recharge \\
Transition & transition \\
Stabilité et insensibilité & enrochement \\
à l'eau & \\
\hline
\end{tabular}

\subsection{Résistance fonction de classe}

Les tableaux 2 et 3 montrent la répartition de $\Phi$ pour trois classes de matériaux au sein du barrage. Deux types de données concernant langle de frottement sont disponibles dans la banque:

- celles qui sont liés à $\Delta \Phi$ (tableau 2) ;

- et celles qui sont en dépendance étroite avec la cohésion c (tableau 3).

Sur les deux tableaux, la décroissance de l'angle de frottement interne de l'enrochement jusqu'au noyau avec plus de $50 \%$ de fines, est nette.
Tableau 2. - Evolution de l'angle de frottement suivant la classe du matériau.

$L^{\prime}$ 'angle de frottement à $\sigma_{3}=\mathrm{Pa}=0,1 \mathrm{MPa}$ correspondant à l'expression de $\Phi_{1}$ donnée par :

$$
\Phi\left(\sigma_{3}\right)=\Phi_{1}-\Delta \Phi \log _{10}\left(\frac{\sigma_{3}}{\mathrm{~Pa}_{\mathrm{a}}}\right)
$$

\begin{tabular}{|c|c|c|c|c|c|c|}
\hline \multirow{2}{*}{ Classe } & \multicolumn{3}{|c|}{$\Phi_{1}\left({ }^{\circ}\right)$} & \multicolumn{3}{|c|}{$\operatorname{tg} \Phi_{1}$} \\
\hline & $\begin{array}{c}\text { moyenne } \\
\mathrm{m}\end{array}$ & $\begin{array}{c}\text { ecart-type } \\
a\end{array}$ & $\sigma / m$ & m & $\sigma$ & $\sigma / m$ \\
\hline Enrochement & 45,91 & 5,21 & 0,11 & 1,05 & 0,21 & 0,20 \\
\hline Filtre & 39,83 & 5,63 & 0,14 & 0,85 & 0,18 & 0,21 \\
\hline (f $\begin{array}{c}\text { noyau } \\
<50 \% \text { ) }\end{array}$ & 36,39 & 7,78 & 0,21 & 0,75 & 0,20 & 0.26 \\
\hline (f $>50 \%)$ & 30,00 & 2,30 & 0,08 & 0,58 & 0,04 & 0,06 \\
\hline
\end{tabular}

On remarque sur le tableau 3 que l'angle de frottement lié à la cohésion est plus faible par rapport au cas où cet angle est lié à $\Delta \Phi$ et ceci pour toutes les classes de matériaux. 
Tableau 3. - Evolution de l'angle de frottement suivant la classe du matériau.

L'angle de frottement correspondant à l'expression de $\Phi_{1}$ donnée par :

$$
\tau=c+\sigma_{n} \operatorname{tg} \Phi
$$

\begin{tabular}{|c|c|c|c|c|c|c|}
\hline \multirow{2}{*}{ Classe } & \multicolumn{3}{|c|}{$\Phi_{1}\left({ }^{\circ}\right)$} & \multicolumn{3}{|c|}{ tg $(\Phi)$} \\
\cline { 2 - 7 } & $\begin{array}{c}\text { moyenne } \\
\mathrm{m}\end{array}$ & $\begin{array}{c}\text { écart-type } \\
\sigma\end{array}$ & $\sigma / \mathrm{m}$ & $\mathrm{m}$ & $\sigma$ & $\sigma / \mathrm{m}$ \\
\hline $\begin{array}{c}\text { Enrochement } \\
\text { Filtre } \\
\text { noyau }\end{array}$ & $\begin{array}{l}42,00 \\
\text { (f }<50 \% \text { ) }\end{array}$ & 28,94 & 0,07 & 0,90 & 0,09 & 0,10 \\
$\begin{array}{c}\text { noyau } \\
\text { (f }>50 \% \text { ) }\end{array}$ & 24,67 & 8,52 & 0,10 & 0,75 & 0,11 & 0,14 \\
& 8,55 & 0,34 & 0,47 & 0,18 & 0,38 \\
\hline
\end{tabular}

La décroissance de l'angle de frottement avec la contrainte est d'autant plus grande que le matériau est grossier, comme le montre le tableau 4 . Elle varie de 9 degrés pour les enrochements, à 2 degrés pour les argiles fines du noyau. On note deux types de noyaux : ceux en argile et ceux en sable et grave argileux. La frontière entre les deux est $\Phi=30^{\circ}$.

L'expression :

$$
\Phi_{10} / \Phi_{1}=\left(\Phi_{1}-\Delta \Phi\right) / \Phi_{1}
$$

du tableau 4 mesure la variation relative de $\Phi$ entre $0,1 \mathrm{MPa}$ et $1 \mathrm{MPa}$.

\subsection{Déformabilité des matériaux}

Le module $\mathrm{K}$ varie considérablement suivant Pemplacement des matériaux. Les enrochements et les drains ont des valeurs moyennes voisines de 800 , alors que les noyaux sableux et graveleux descendent à 330 , et les noyaux d'argile fine atteignent la valeur de 50 (tableau 5).

Ce résultat met en évidence le risque de fissuration des barrages en enrochement à noyau argileux.
Tableau 4 - Décroissance de $\Delta \Phi$ de la recharge vers le noyau.

\begin{tabular}{|c|c|c|c|c|c|}
\hline \multirow{2}{*}{ Classe } & \multicolumn{3}{|c|}{$\Phi_{1}\left({ }^{\circ}\right)$} & \multicolumn{2}{c|}{$\left(\Phi_{1}-\Delta \Phi\right) / \Phi_{1}$} \\
\cline { 2 - 6 } & $\begin{array}{c}\text { moyenne } \\
\mathrm{m}\end{array}$ & $\begin{array}{c}\text { écart-type } \\
\sigma\end{array}$ & $\mathrm{m}$ & $\sigma$ & $\sigma / \mathrm{m}$ \\
\hline $\begin{array}{c}\text { Enrochement } \\
\text { Filtre }\end{array}$ & 9,6 & 4,0 & 0,85 & 0,11 & 0,13 \\
$\begin{array}{c}\text { noyau } \\
<50 \%)\end{array}$ & 6,8 & 5,7 & 0,81 & 0,07 & 0,08 \\
$(f<50$ & 2,8 & 0,79 & 0,11 & 0,14 \\
\hline
\end{tabular}

La croissance du module avec la contrainte est cependant d'autant plus forte que le pourcentage de fines augmente. Le tableau 5 montre un exposant $n$ compris entre 0,25 et 0,4 pour les matériaux grossiers, et un exposant supérieur à 0,6 pour les matériaux de noyau. Ce phénomène est heureusement bénéfique car il diminue la déformabilité différentielle entre le noyau et les filtres ou drain qui reste cependant très forte pour les barrages de taille modeste.

L'écart-type du module $\mathrm{K}$ est très grand, car des valeurs très fortes ont été répertoriées. Cette dispersion est à la fois symptomatique de la difficulté de mesurer le module et de sa grande variabilité.

Le paramètre Rf présenté dans le tableau 5 , est en moyenne soit 0,7 (enrochement, transition, recharges), soit 0,8 (filtre et drain).

Le tableau 6 donne une idée des valeurs de G, F et $\mathrm{D}$ pour les différents emplacements du barrage. On y note toutefois une valeur moyenne de $G$ variant de 0,30 à 0,35 , et une valeur moyenne de $D$ qui varie de 6,5 à 9 .

\section{INFLUENCE CONJUGUÉE DE LA GRANULOMÉTRIE ET DE L'INDICE DES VIDES}

\subsection{Densité fonction de la granulométrie}

La densité des échantillons ne devrait en fait dépendre principalement que de la granulométrie. En effet,

Tableau 5. - Evolution des paramètres définissant le module d'YOUNG en fonction des classes.

\begin{tabular}{|c|c|c|c|r|r|r|}
\hline \multirow{2}{*}{ Classe } & \multicolumn{2}{|c|}{ Rf } & \multicolumn{2}{c|}{ K } & \multicolumn{2}{c|}{$\mathrm{n}$} \\
\cline { 2 - 7 } & moyenne m & écart-type $\sigma$ & $\mathrm{m}$ & $\sigma$ & $\mathrm{m}$ & $\sigma$ \\
\hline Enrochement & 0,71 & 0,10 & 813 & 691 & 0,40 & 0,21 \\
Recharge & 0,71 & 0,15 & 762 & 793 & 0,50 & 0,22 \\
Transition & 0,71 & 0,10 & 1225 & 1041 & 0,25 & 0,46 \\
Drain & 0,85 & 0,10 & 860 & 589 & 0,28 & 0,21 \\
Filtre & 0,81 & 0,11 & 941 & 583 & 0,46 & 0,21 \\
Noyau (f $<50 \%)$ & 0,80 & 0,12 & 340 & 247 & 0,60 & 0,35 \\
Noyau (f $>50 \%)$ & 0,69 & 0,10 & 50 & 40 & 0,70 & 0,10 \\
\hline
\end{tabular}


Tableau 6. - Evolution des paramètres définissant le coefficient de Poisson en fonction des classes.

\begin{tabular}{|c|c|c|c|c|c|c|}
\hline \multirow{2}{*}{ Classe } & \multicolumn{2}{|c|}{ G } & \multicolumn{2}{|c|}{$\mathrm{F}$} & \multicolumn{2}{|c|}{ D } \\
\hline & moyenne $\mathrm{m}$ & ecart-type $\sigma$ & $\mathrm{m}$ & $\sigma$ & $\mathrm{m}$ & $\sigma$ \\
\hline $\begin{array}{c}\text { Enrochement } \\
\text { Recharge } \\
\text { Transition } \\
\text { Drain } \\
\text { Filtre } \\
\text { Noyau (f }<50 \% \text { ) }\end{array}$ & $\begin{array}{l}0,34 \\
0,33 \\
0,32 \\
0,30 \\
0,35 \\
0,32\end{array}$ & $\begin{array}{l}0,08 \\
0,11 \\
0,08 \\
0,06 \\
0,03 \\
0,12\end{array}$ & $\begin{array}{l}0,16 \\
0,10 \\
0,14 \\
0,28 \\
0,16 \\
0,10\end{array}$ & $\begin{array}{l}0,10 \\
0,10 \\
0,08 \\
0,15 \\
0,10 \\
0,10\end{array}$ & $\begin{array}{l}6,69 \\
7,13 \\
8,95 \\
7,30 \\
6,52 \\
7,28\end{array}$ & $\begin{array}{r}3,66 \\
3,42 \\
6,34 \\
3,81 \\
3,02 \\
14,34\end{array}$ \\
\hline
\end{tabular}

la plupart des matériaux sont compactés à l'énergie proche du Proctor Normal. Ainsi l'indice des vices devrait suivre l'évolution définie par BIAREZ et
ROPERS (1980) en fonction de l'étalement de la granulométrie et de la forme des grains, mais l'on note des variations importantes (fig. 2).

\section{BARRAGES EN ENROCHEMENT}

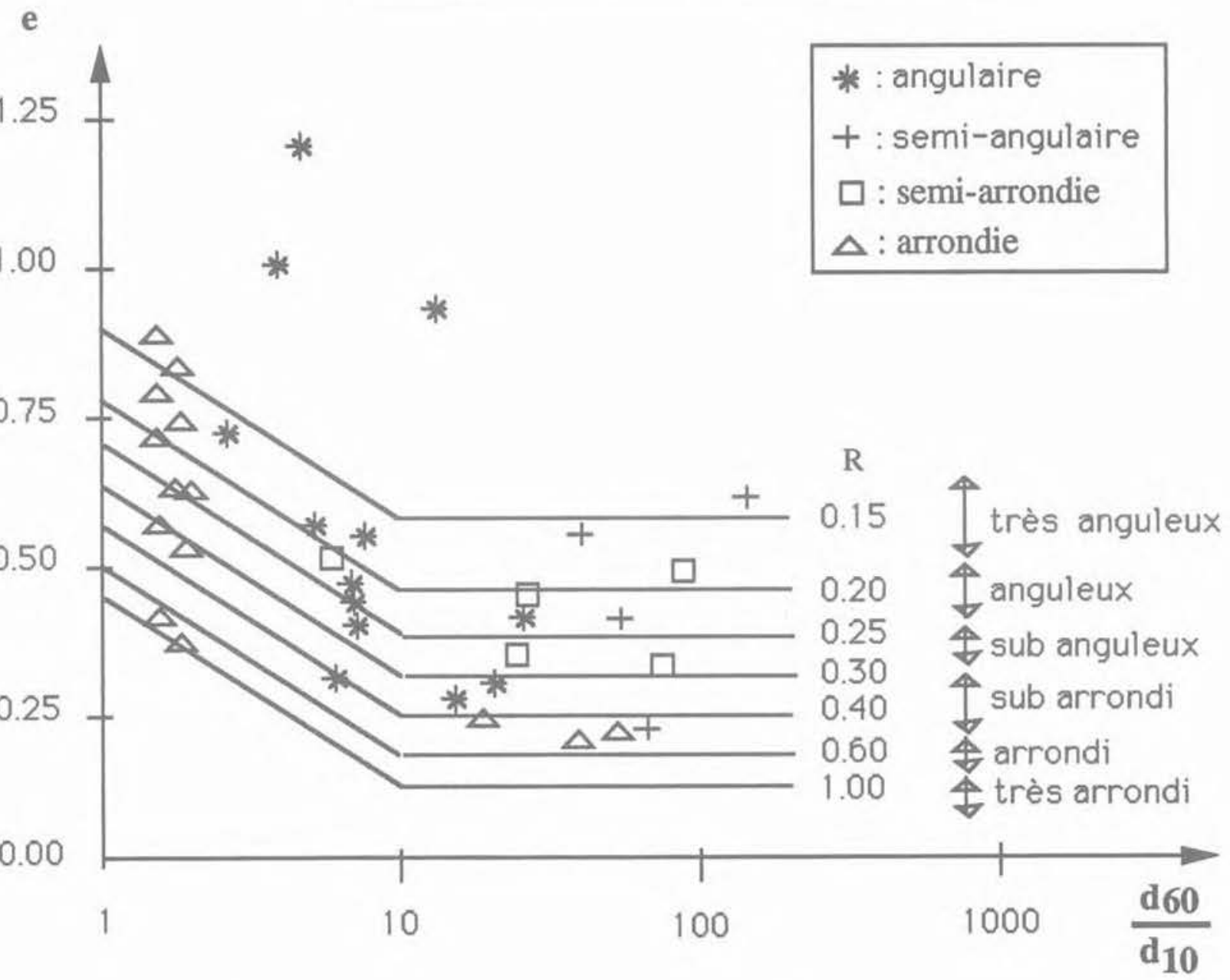

Fig. 2. - Variation d'indice des vides en fonction de $\frac{D_{60}}{d_{10}}$ et de l'angularité

(sols avec moins de $10 \%$ de fines ( $d_{10}<80$ microns)). Les droites proviennent de l'analyse de ROPERS. 
Au-delà de $\mathrm{d}_{60} / \mathrm{d}_{10}=15$, l'indice des vides ne devrait dépendre que de l'angularité ; en fait on constate seulement que les particules arrondies créent les arrangements les plus denses.

\subsection{Résistance}

\section{a. Le rôle de l'indice des vides}

La figure 3 représente la variation de l'angle de frottement interne du noyau, du filtre et de l'enrochement, avec l'indice des vides après compactage. Il semblerait que lindice des vides n'ait d'influence significative que sur les matériaux du noyau, car en augmentant, il fait chuter l'angle de frottement.

\section{b. Le rôle du pourcentage de fines}

La figure 4 montre une décroissance linéaire de l'angle de frottement avec le pourcentage des fines. Cette tendance est confirmée malgré une dispersion importante.

c. Le rôle de la limite de liquidité $W_{L}$

Nous observons sur la figure 5 que l'angle de frottement décroît quand $W_{L}$ augmente. Ce graphique prend en compte les matériaux ayant une valeur de $\mathrm{d}_{10}<20$ microns (en fait ce graphique reste presque inchangé pour $\mathrm{d}_{10}<80$ microns). Nous vérifions que :

- la plupart des points se situent au dessus de la courbe $\Phi_{\mathrm{pp}}$ de l'angle de plasticité parfaite (TAIBI) ; - la courbe de langle de frottement résiduel, $\Phi$ résiduel, se trouve encore plus en dessous de $\Phi_{\mathrm{pp}}$. $\mathrm{Ce}$ qui est conforme au fait que les résistances de cette banque ne correspondent pas aux $\Phi$ résiduels, mais au pic ;

- il existe un point particulier (point entouré) qui correspond au matériau du noyau du barrage de Mrica (d'une hauteur de $110 \mathrm{~m}$, situé à Java, en Indonésie) dont quelques caractéristiques sont données au tableau 7.

La faible valeur de K (50) et un angle de frottement fort pour un noyau $\left(30^{\circ}\right)$ font de ce matériau un matériau particulier, d'autant plus que sa valeur de $\mathrm{W}_{\mathrm{L}}=84 \%$ est une valeur extrême de la banque de données. En fait ce matériau est représentatif des sols résiduels andosols et autres argiles riches en halloïsite qui possèdent une grande résistance malgré des limites d'Atterberg importantes et une grande déformabilité.

\section{BARRAGES EN ENROCHEMENT}

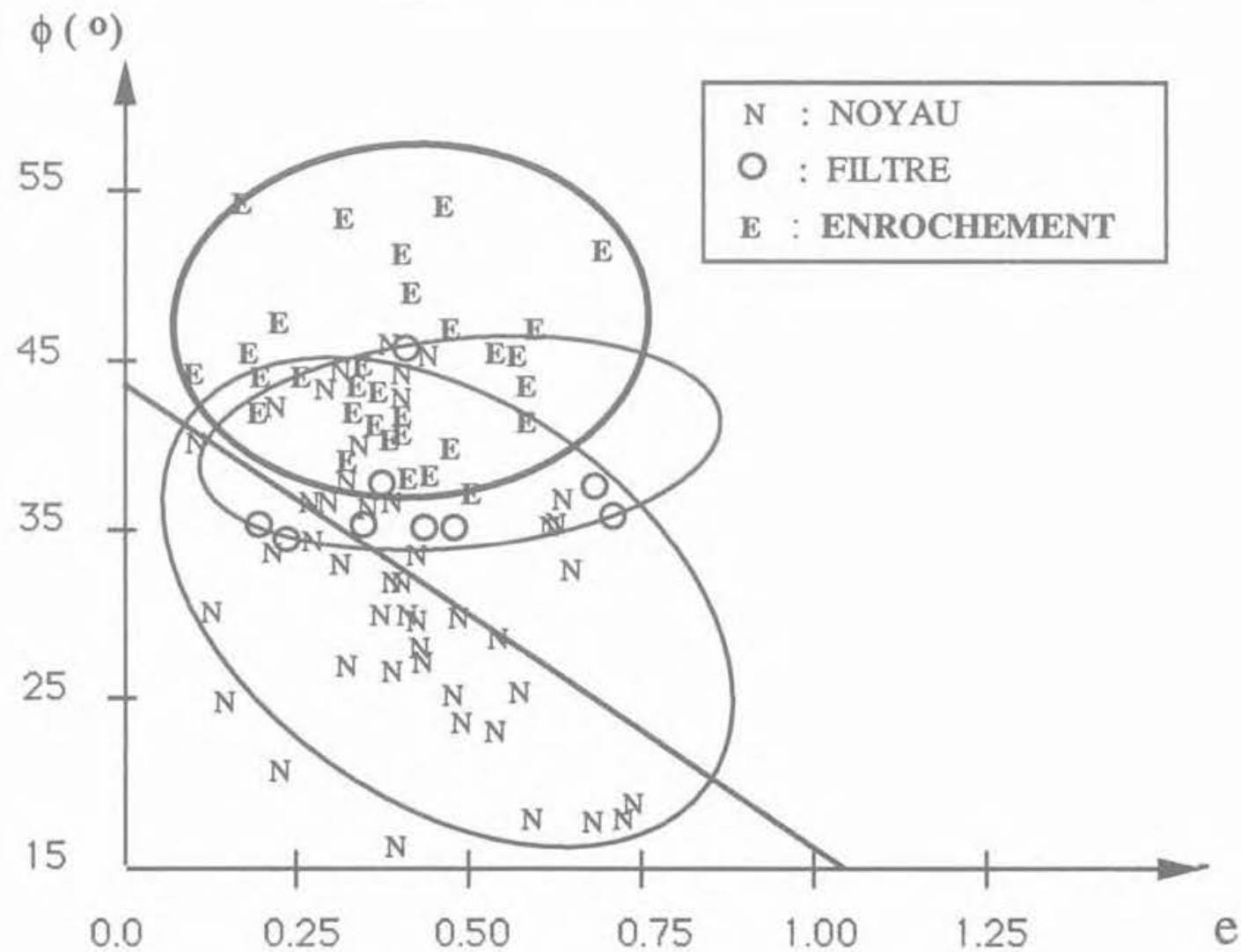

Fig. 3. - Variation de $\Phi$ en fonction de l'indice des vides. 
$\phi\left({ }^{0}\right)$

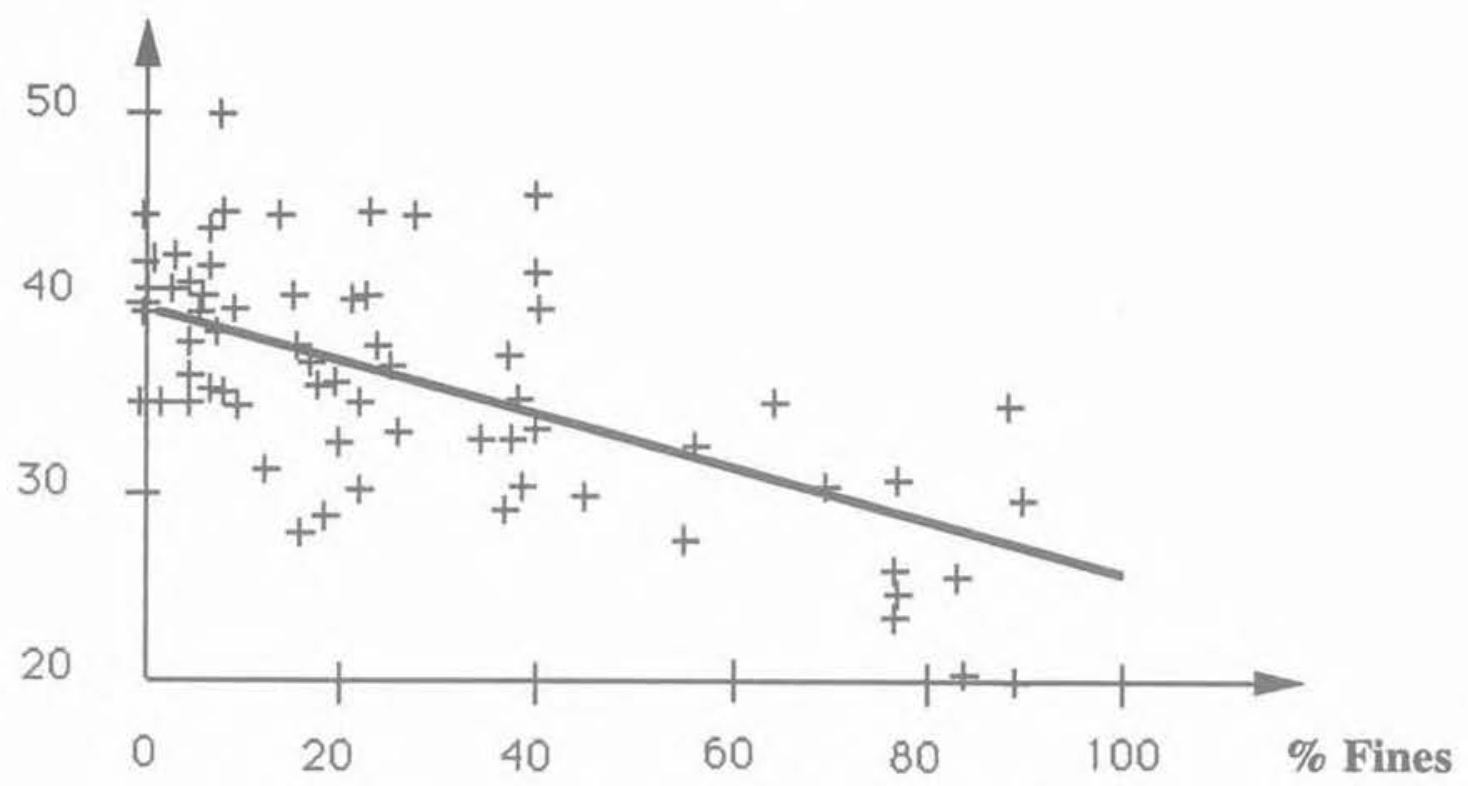

Fig. 4. - Variation de $\Phi$ en fonction de pourcentage de fines (tous les matériaux du barrage).

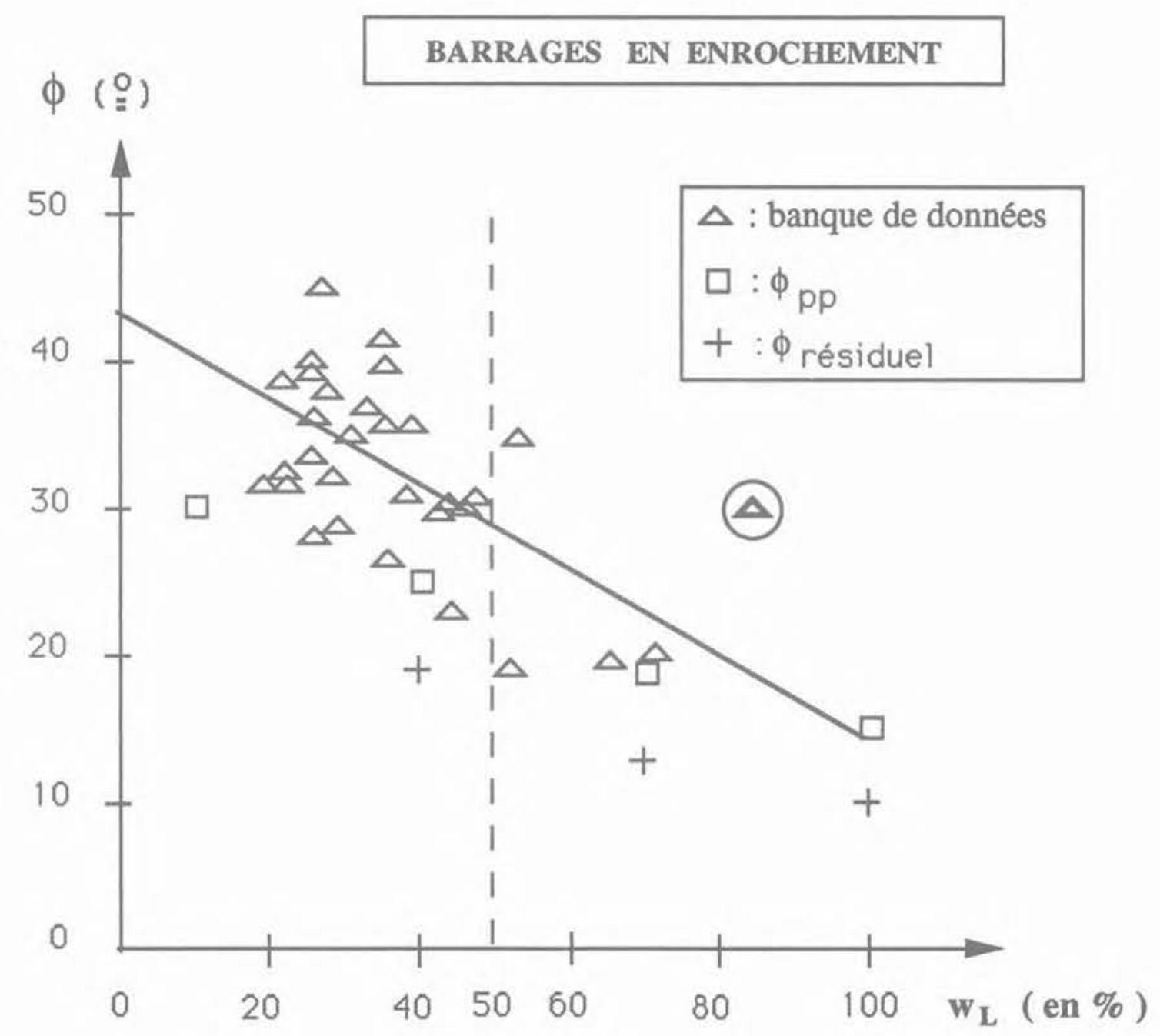

Fig. 5. - Variation de $\Phi$ en fonction de $W_{L}$. (Les matériaux avec $d_{10}<20$ microns). 
Tableau 7. - Caractéristiques du matériau de noyau du barrage de Mrica.

\begin{tabular}{|c|c|c|c|c|c|c|c|c|}
\hline \multicolumn{4}{|c|}{ Caractéristiques du sol } & \multicolumn{4}{c|}{ Paramètres de DUNCAN } \\
\hline Type du sol & $W_{\mathrm{L}}$ & $\mathrm{W}$ & $c$ (MPa) & $\Phi$ & Kur & $K$ & $\mathrm{n}$ & $\mathrm{Rf}$ \\
\hline Argile halloïsite & 84 & 57,5 & 0,01 & 30 & 150 & 50 & 0,7 & 0,69 \\
\hline
\end{tabular}

\section{d. Le rôle de la taille des grains}

Il est intéressant de noter une évolution en forme de cloche (fig. 6) qui laisserait supposer qu'au-delà d'un certain diamètre, l'angle de frottement diminue (à l'échelle d'un milieu continu). C'est sans doute parce que pour $\mathrm{d}_{10}$ grand $e$ est grand aussi.

L'angle de frottement maximal est constaté pour $\mathrm{d}_{10}$ $=1 \mathrm{~mm}$.

e. Paramètre e.tg $\Phi$ en fonction de $d_{60} / d_{10}$ et de l'angularité

De même, sur la figure 7 nous avons remplacé e par e.tg $\Phi$ pour voir son évolution en fonction de $\mathrm{d}_{60} / \mathrm{d}_{10}$. Nous voyons les allures de courbes sembla- bles à celles de e fonction de $\mathrm{d}_{60} / \mathrm{d}_{10}$. Nous vérifions un résultat intéressant annoncé par AL-ISSA pour le sable d'Hostun, à savoir e. $\operatorname{tg} \Phi=0,5$. En fait il existe une légère décroissance avec $\mathrm{d}_{60} / \mathrm{d}_{10}$ jusqu'à $\mathrm{d}_{60} / \mathrm{d}_{10}$ $>15$. Là, est sous-jacente lidée d'un arrangement optimum des petits grains dans les vides laissés par les gros.

D'autre part, le paramètre e.tg $\Phi$ varie peu pour un sol donné et il dépend non seulement de $\mathrm{d}_{60} / \mathrm{d}_{10}$ et de l'angularité, mais également de la minéralogie du sol en question.

L'intérêt de cette expression est de déterminer, pour certains types de matériaux, la variation d'angle de frottement en fonction du compactage.

.

\section{BARRAGES EN ENROCHEMENT}

$\phi\left(0^{\circ}\right)$

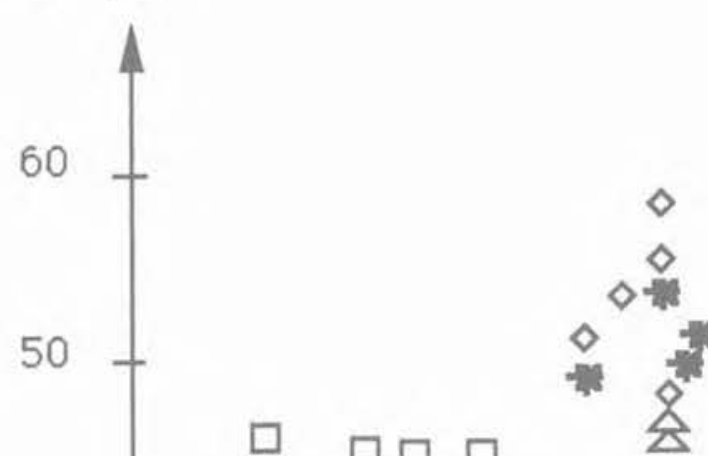

$\Delta$ : filtre

: noyau

+ : drain

$\diamond:$ transition

- enrochement

40

40
20<smiles></smiles>

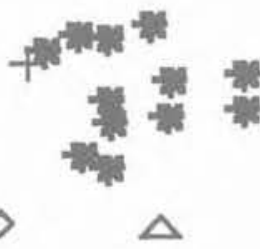

$\triangle{ }_{\Delta} \Delta \Delta$

$\triangle$

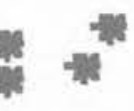




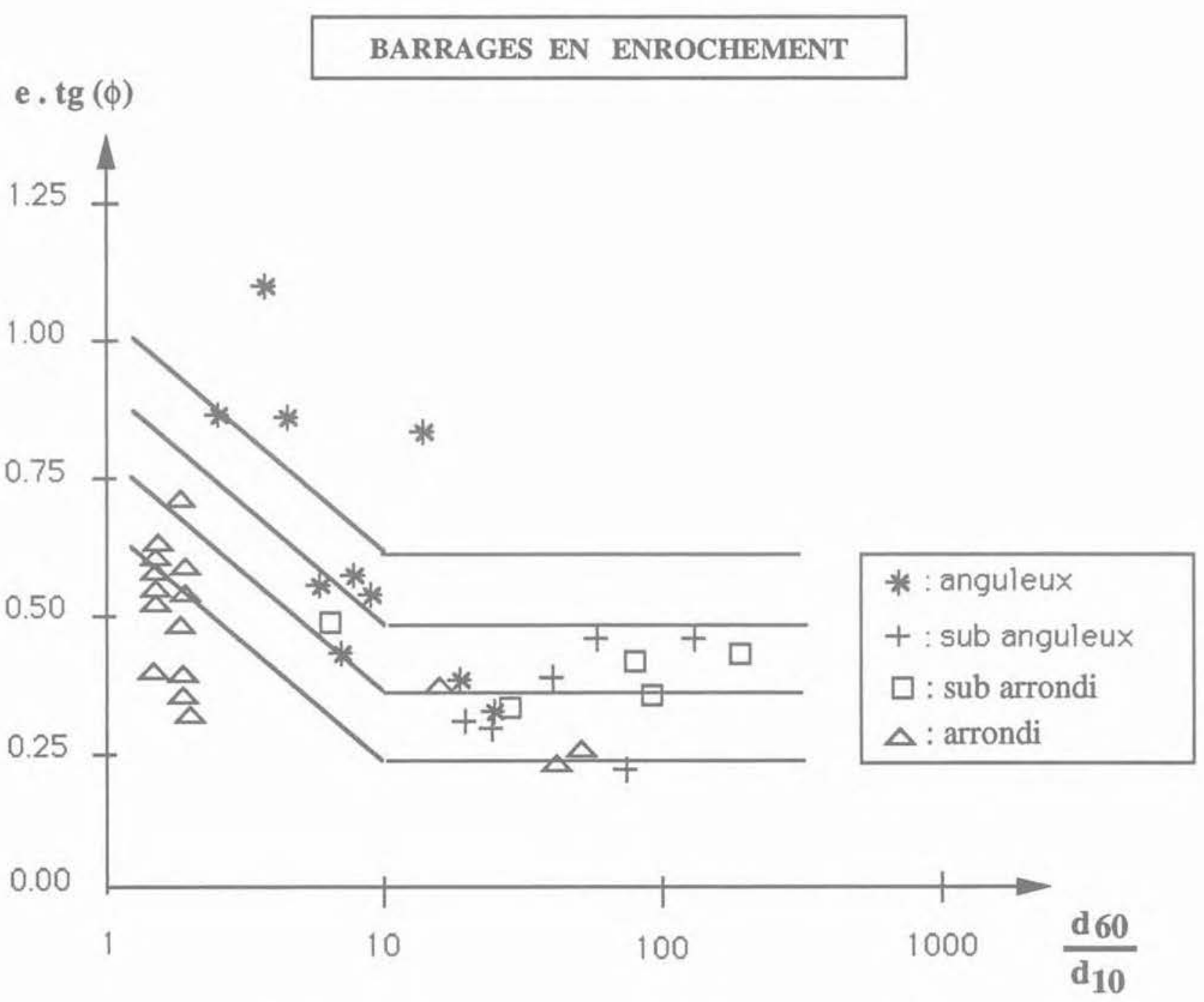

Fig. 7. - Hypothèse sur la variation de e.tg $(\Phi)$ en fonction de $\frac{d_{60}}{d_{10}}$
et l'angularité (sols avec moins de $10 \%$ de fines $(d, 10<80$ microns).

\subsection{Déformabilité}

\section{a. Le rôle de l'indice des vides}

La figure 8 montre la baisse linéaire du module $\mathrm{K}$ avec l'indice des vides. Cette décroissance s'effectue de manière similaire pour le noyau d'une part, le filtre, le drain et la transition d'autre part.

La dispersion des valeurs de $\mathrm{n}$ dans la banque était telle que nous ne pouvions rien conclure sur la variation de $n$ en fonction des vides pour l'ensemble des matériaux. Par contre, nous tirons profit du classement par catégorie de ces matériaux, cormme nous le montre la figure 9.

La croissance est assez forte pour le noyau. En revanche, l'enrochement a un exposant décroissant qui traduit une tendance à module constant. En effet, après une rigidité initiale forte à moyenne d'enrochement, l'écrasement des particules limites la rigidificaiton du matériau.
On remarque sur la figure 9 que pour des faibles valeurs d'indice des vides, la valeur de $n$ est non loin de 0,5 pour le noyau et l'enrochement.

\section{b. Le rôle de $d_{10}$}

La figure 10 représente la variation de $\mathrm{K}$ en fonction de $d_{10}$. On remarque deux zones distinctes:

- dans la première zone où $\mathrm{d}_{10}<0,4 \mathrm{~mm}, \mathrm{~K}$ croît avec $\mathrm{d}_{10}$;

- dans la zone où $\mathrm{d}_{10}>0,4 \mathrm{~mm}, \mathrm{~K}$ décroît.

Mais on voit qu'il $y$ a des valeurs de $\mathrm{K}$ entre :

$0,1 \mathrm{~mm}<\mathrm{d}_{10}<1 \mathrm{~mm}(100<\mathrm{K}<4000)$ qui sont supérieures à celles des autres zones :

$$
(100<\mathrm{K}<1000) \text {. }
$$

Ce graphique nous amène aux remarques suivantes: - pour les matériaux d'enrochement, drain, filtre et transition (matériaux grossiers), $\mathrm{K}$ décroît avec $\mathrm{d}_{10}$. 
K

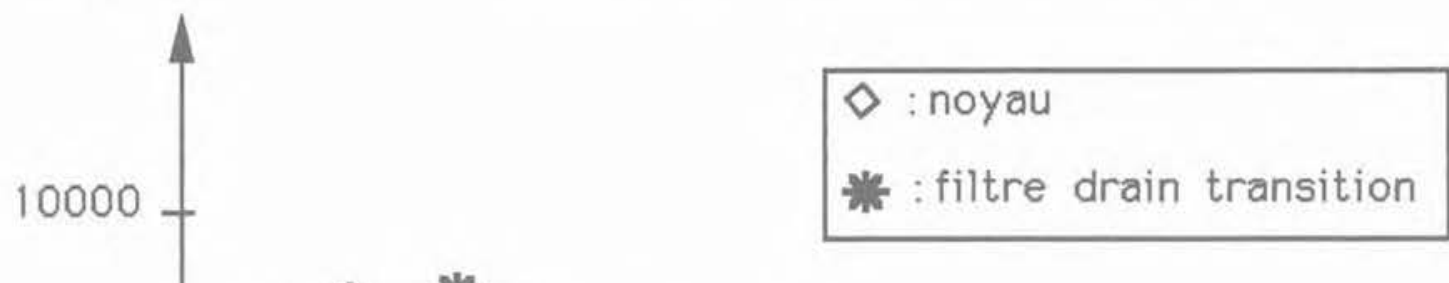

1000

100

10

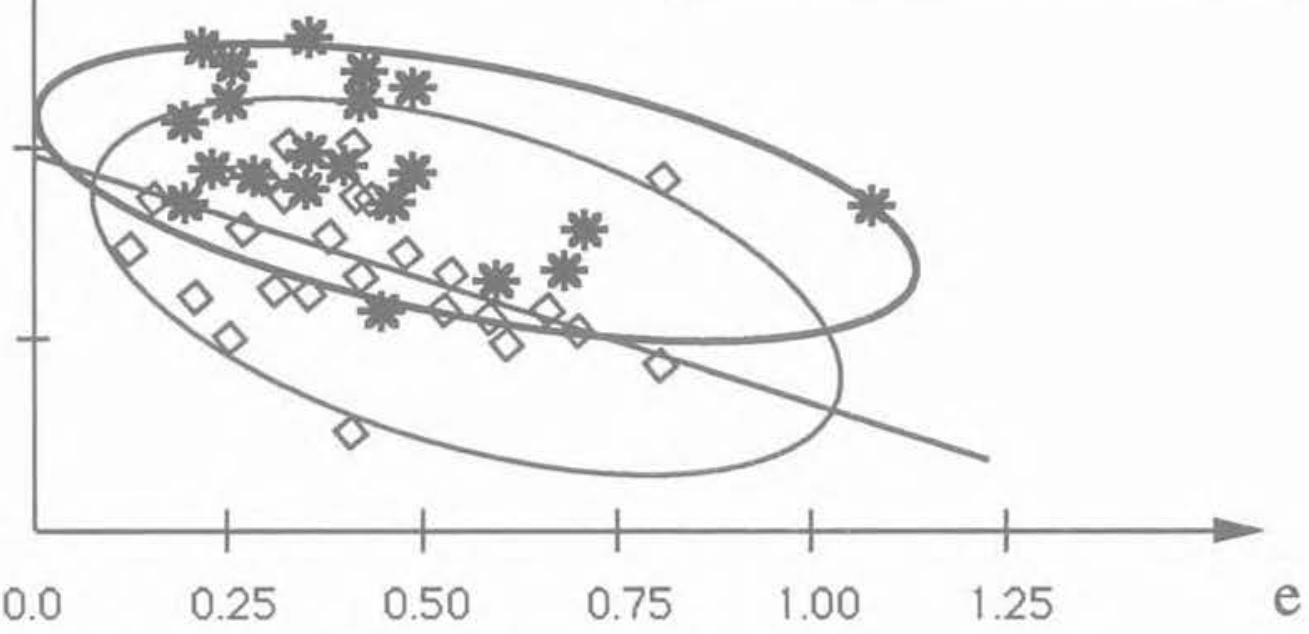

Fig. 8. - Variation du module $K$ en fonction de l'indice des vides.

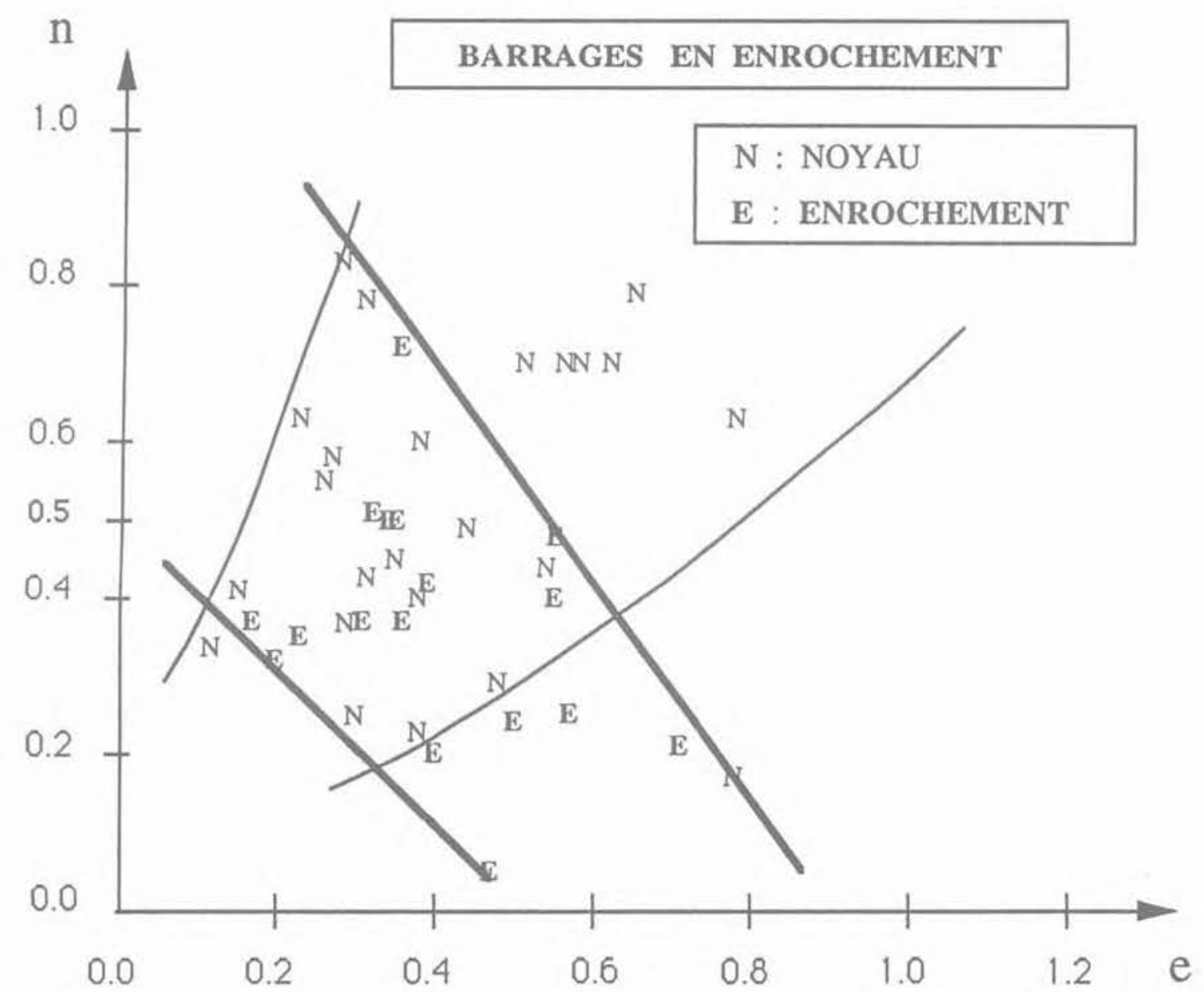

Fig. 9. - Variation de $n$ en fonction de l'indice des vides. 
$\mathbf{K}$

BARRAGES EN ENROCHEMENT

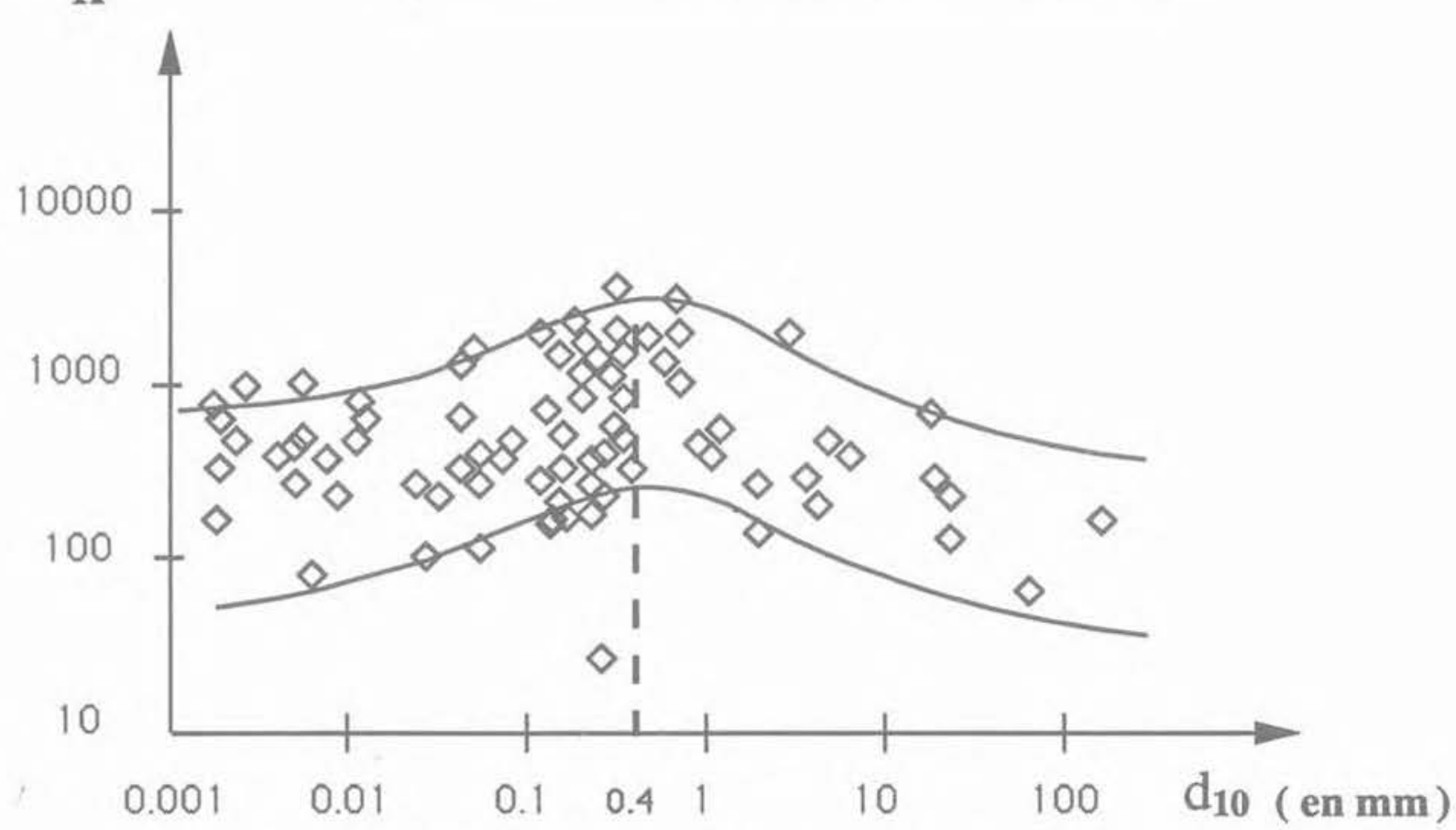

Fig. 10. - Variation du module $K$ en fonction de $d_{10}$ (tous les matériaux).

Cette décroissance est due à l'angularité des matériaux et aussi au manque de fines ;

- en revanche, $\mathrm{K}$ croît avec $\mathrm{d}_{10}$ pour les matériaux fins comme celui du noyau. Nous savons en effet que le module de Young $\mathrm{E}$ diminue avec le pourcentage de fins.

\section{CONCLUSION}

Quelques phénomènes essentiels concernant le comportement des sols ont été retrouvés et chiffrés par exploitation statistique de cette banque.

L'intérêt majeur de cette banque est de fournir un échantillon de paramètres suffisamment étoffés sur des matériaux difficiles à mesurer, pour en connaître leur ordre de grandeur et leur domaine de variation, ainsi que d'identifier le sens de variation de chaque paramètre en fonction des autres.

Cependant, si ces grandes tendances sont utiles pour des études d'avant-projet, elles sont à affiner par une analyse de cas, afin de réduire une importante dispersion.

\section{BIBLIOGRAPHIE}

AL-ISSA M. (1973), Recherche de lois contraintedéformation des milieux pulvérulents. Analyse de la validité des lois hyperboliques. Application aux pieux et barrages. Thèse de Docteur Ingénieur, ECP.
BIAREZ J., HICHER P.Y. (1990), Lois de comportement des sols remaniés et des matériaux granulaires. Notes de cours (tome 1) ECP.

BIAREZ J., TAIBI S. (1991), Classement des paramètres en mécanique des sols. Séminaire ECP, 18-22 novembre.

CHANG C.Y., DUNCAN J.M. (1970), Analysis of soil movement around a deep excavation. Proc. ASCE Vol. 96, n SM5.

CHARLES J.A., SOARES M.M. (1984), Stability of compacted rockfill slopes, Géotechnique, 34, p. $61-70$.

FRY J.J., FLAVIGNY E., MAMBA M. (1989), Classification et propriétés des enrochements : cas d'un grès. Proceeding of the 12th. International Conference on Soil Mechanics and Foundation Engineering, Rio De Janeiro, p. 713-714.

JANSSON S., NILSSON A. (1991), Experience of halloysitic clay in damfills and foundations at the Mrica dam, Indonesia. CIGB 1991, Vienne, Q 61 R 1.

KONDNER R.L. (1963), Hyperbolique stress-strain response: cohesive soils. ASCE. J. Soil Mech. and Found. Div., N० SM1, p. 115-143.

NEDJAT N. (à paraître), Lois de comportement des barrages en enrochement. Thèse de Doctorat, ECP.

ZERVOYANNIS C. (1982), Etude synthétique des propriétés mécaniques des argiles saturées et des sables sur chemin œdométrique et triaxial de révolution. Thèse de Docteur Ingénieur, ECP. 